\title{
Geographical determinants of health
}

\section{Takashi Nakamura*}

Center for Community Medicine, Jichi Medical University, 3311-1 Yakushiji, Shimotsuke, Tochigi, Japan

The enjoyment of the highest attainable standard of health is one of the fundamental rights of every human being without distinction of race, religion, political belief, economic or social condition [1]. Social determinants of health include economic status, educational level, social class, social capital, and geographic conditions. Access to health care is a vital determinant of health and it is one of the fundamental concepts in primary care.

The concepts of geographical access to primary care has been described in past research [2]. Satisfactory availability of primary care is related to better health status, lower overall mortality, lower death rates due to diseases of heart and cancer, longer life expectancy, lower neonatal death rate, and lower cases of low birth weight [3]. These are some of examples of geographical determinants of health.

Several measures of geographical determinants of health have been proposed. One of the simplest measures is the provider-to-population ratio, also called supply ratio. This measure is intuitive and the easiest to understand, and corresponds to "availability" which is a subordinate concept of "access."

supply ratio $=\frac{\text { the number of providers within certain area }}{\text { population at risk within certain area }}$

Travel impedance to the nearest provider is also often used as a measure that is popular in the field of emergency medical care [4]. Distance or travel time are commonly used to measure travel impedance when we are interested in arriving at the nearest health care provider. They correspond to the term "accessibility," which is a subordinate concept of "access."

travel impedance $=$ minimum $($ distances between population and provider $)$

Complex measures such as the enhanced two-step floating catchment area (E2SFCA) method [5] or its variance are also used. In the first step of E2SFCA, the provider-to-population ratio for each provider is calculated. The population at risk is identified according to the impedance between provider and population center. In the second step, the provider-to-population ratio for each provider is summed up within the specific impedance from each population center. Some variations of floating catchment area methods have been proposed with different calculation of impedance. Nakamura et al. showed the regional differences in primary care access using an enhanced two-step floating catchment area method [6].

$E 2 S F C A=\sum_{\text {neighbor provider }} \frac{\text { impedance weight } \times \text { provider }}{\sum_{\text {neighbor population }} \text { impedance } \text { weight } \times \text { population }}$

Some other measures such as gravity model, migration preference index, and Kernel density methods have also been proposed. Each measure has its pros and cons. Detailed evaluation of these measures has not been done.

The development of geographic information systems supports the measures of geographical determinants of health. Thus, the application of geographic information systems to the healthcare field has attracted attention [7].

In practicing community-oriented primary care, it is indispensable to understand the community. The geographical determinants of health could help us improve the quality of community medicine.

\section{References}

1. WHO (2005) Constitution of the World Health Organization. World health organization.

2. Guagliardo MF (2004) Spatial accessibility of primary care: concepts, methods and challenges. Int J Health Geogr 3: 3. [Crossref]

3. Shi L (1994) Primary care, specialty care, and life chances. Int J Health Serv 24: 431458. [Crossref]

4. Miwa M, Kawaguchi H, Arima H, Kawahara K (2006) The effect of the development of an emergency transfer system on the travel time to tertiary care centres in Japan. Int $J$ Health Geogr 5: 25 .

5. Luo W, Qi Y (2009) An enhanced two-step floating catchment area (E2SFCA) method for measuring spatial accessibility to primary care physicians. Health Place 15: 1100.

6. Nakamura T, Nakamura A, Mukuda K, Harada M, Kotani K, et al. (2017) Potential accessibility scores for hospital care in a province of Japan: GIS-based ecological study of the two-step floating catchment area method and the number of neighborhood hospitals. BMC Health Serv Res 17: 438.

7. Graves BA (2008) Integrative literature review: a review of literature related to geographical information systems, healthcare access and health outcomes. Perspect Health Inf Manag 5: 11.
Copyright: (C2018 Nakamura T. This is an open-access article distributed under the terms of the Creative Commons Attribution License, which permits unrestricted use, distribution, and reproduction in any medium, provided the original author and source are credited.
Correspondence to: Takashi Nakamura, Center for Community Medicine, Jichi Medical University, 3311-1 Yakushiji, Shimotsuke, Tochigi, Japan, Tel: +81-28558-7498; E-mail: nakamurata@jichi.ac.jp

Received: February 04, 2018; Accepted: February 26, 2018; Published: February 28,2018 